\title{
Development and Experiment on Digital Pre-Distortion for Analog Beamforming Systems
}

\author{
Haeng-Bok Kil, Kwang-Pyo Lee, Sung-Mi Jo and Eui-Rim Jeong* \\ Department of Communication and Information Engineering, Hanbat National \\ University, \\ erjeong@hanbat.ac.kr(*corresponding author)
}

\begin{abstract}
This paper proposes a digital pre-distortion (DPD) technique for analog beamforming systems. The considering system has M PAs ( $M$ is the number of antennas) and one digital chain. In contrast to the conventional DPD problem where one DPD linearizes one PA, a single DPD should linearize M PAs simultaneously in the analog beamforming system. In this paper, we design a new DPD by minimizing the sum of linearization errors of M PAs. To verify the proposed DPD, computer simulation and experiment are conducted. The results confirm that the proposed DPD can successfully linearize the multiple PAs in analog beamforming systems.
\end{abstract}

Keywords: Digital Pre-Distortion (DPD); Analog beamforming; Adaptive algorithm; Power amplifier; Polynomial

\section{Introduction}

MIMO (multiple input multiple output) techniques based on multiple antennas have been widely used these days [1]-[4]. While MIMO systems significantly improve the multiplexing and diversity gains in wireless communications, they require larger cost and size due to multiple signal paths. Those burdens increase as the number of antennas grow. Among MIMO techniques, analog beamforming (BF) has relatively low cost and small size since it is composed of one digital chain, $M$ phase shifters, power amplifiers (PAs) and antennas, where $M$ is the number of antennas. In general, PAs have non-linearity, which causes spectral regrowth and inband signal distortion. To mitigate these problems, many linearization techniques have been studied. Among them, the DPD technique is known for excellent linearization performance. The DPD technique compensates for non-linearity of PAs by designing the combined characteristic of DPD and PA linear [5] [6].

This paper proposes a digital pre-distortion (DPD) technique for analog BF systems. The main difficulty for the conventional DPD to be applied to the analog BF systems is that the single DPD must linearize multiple PAs simultaneously since the system has only one digital chain. To design such DPD, we introduce a cost function based on the sum of the square errors for all the PAs. Specifically, the proposed DPD technique combines all the feedback samples from multiple PAs after phase de-rotation, and then the conventional indirect learning method finds the DPD parameters. The computer simulation and experiment results show that the proposed DPD can successfully linearize multiple PAs simultaneously in the analog BF systems.

\section{System Model}

Figure 1 shows the baseband equivalent system model for the analog BF transmitter. The transmitter comprises one digital chain and $M$ antennas. The analog 
BF coefficients are multiplied with the PA inputs by using the phase shifters. The output of $m$-th PA, denoted by $z_{m}(n)$, is $\varphi_{m}\left(\exp \left(j \phi_{m}\right) y(n)\right)$ where $\phi_{m}$ is the rotation phase of $m$-th antenna and $\exp \left(j \phi_{m}\right) y(n)$ is the input of $m$-th PA. The output of DPD, $y(n)$, is $\psi(x(n))$ where $x(n)$ is the transmitted signal. $\varphi(\cdot)$ and $\psi(\cdot)$ are the characteristic functions of PA and PD, respectively.

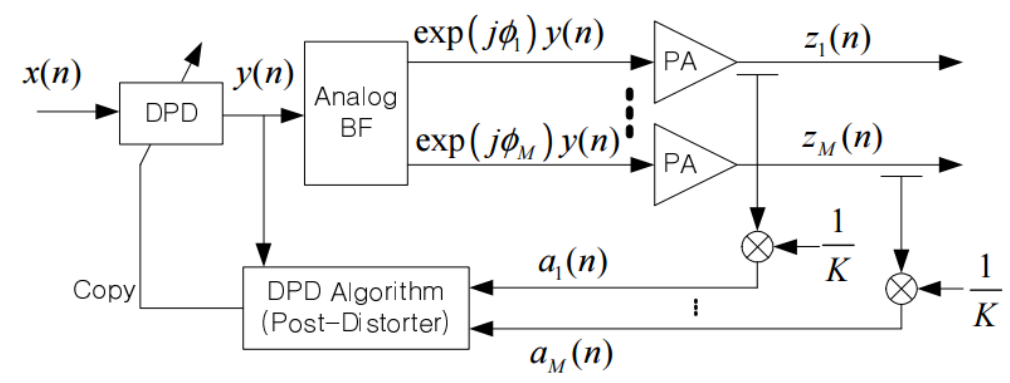

Figure 1. Digital Pre-Distortion Structure in Analog Beamforming Systems

As shown in Figure 1, one DPD must linearize $M$ PAs simultaneously. If the characteristics of PAs are completely the same, constructing one feedback path among $M$ PAs is enough to linearize all the PAs. However, in practice, the PAs have different characteristics. Hence, we need a new DPD strategy considering all the PAs. Assuming that the PA is modeled by a memory polynomial, the $m$-th feedback signal can be written as

$$
\begin{aligned}
& a_{m}(n)=\frac{\varphi_{m}\left(e^{j \phi m} y(n)\right)}{K} \\
& =\sum_{l=0}^{L_{a}} \sum_{p=1}^{P} h_{l, p, m}\left|e^{j \phi m} y(n-l)\right|^{2(p-1)} e^{j \phi m} y(n-l) \\
& =e^{j \phi m} \sum_{l=0}^{L_{a}} \sum_{p=1}^{P} h_{l, p, m}|y(n-l)|^{2(p-1)} y(n-l)
\end{aligned}
$$

where $K$ is the PA gain, $L_{a}$ is the memory depth, and $2 P-1$ is the maximum nonlinear order of the PA. We introduce memory polynomial model for characterizing the DPD function as well. The DPD output can be expressed as

$$
\begin{aligned}
& y(n)=\psi(x(n)) \\
& =\sum_{l=0}^{L_{p}} \sum_{q=1}^{Q} w_{l, q}^{*}|x(n-l)|^{2(q-1)} x(n-l) \\
& =\mathbf{w}^{H} \mathbf{x}(n)
\end{aligned}
$$

where $2 Q-1$ is the maximum polynomial order of DPD, $L_{p}$ is the memory depth, $\mathbf{w}$ is the polynomial coefficient vector given by $\mathbf{w}=$ $\left[w_{0,1}, w_{0,2}, \cdots, w_{0, P}, w_{1,1}, \cdots, w_{Q, L_{P}}\right]^{T} \quad, \quad$ and $\mathbf{x}(n)=\left[x(n), x(n)|x(n)|^{2}, \cdots, x(n)|x(n)|^{2(Q-1)}, x(n-1), \cdots, x\left(n-L_{P}\right) \mid x(n-\right.$ $\left.\left.L_{P}\right)\left.\right|^{2(Q-1)}\right]^{T}$. The number of coefficients to be found is $Q\left(L_{P}+1\right)$. The problem is to find $\mathbf{w}$ that linearizes all the PAs, i.e., $\varphi_{m}\left(e^{j \phi m} \psi(x(n))\right)=K e^{j \phi m} x(n)$ for $m=1, \cdots, M$. 


\section{Proposed DPD}

To design the proposed DPD, the DPD parameters are estimated using an indirect learning method [5]. In this method, the parameters linearizing the PA-postdistorter chain is first found and are then copied to the DPD. In order to design a single postdistorter that linearizes $M$ PA-post-distorter chains, least squares (LS) criterion is used. The cost function is written as

$$
\begin{aligned}
& \boldsymbol{\varepsilon}=\sum_{n=1}^{N} \lambda^{N-n}\left|y(n)-e^{-j \phi_{1}} \mathbf{w}^{H} \mathbf{a}_{1}(n)+\cdots+y(n)-e^{-j \phi_{M}} \mathbf{w}^{H} \mathbf{a}_{M}(n)\right|^{2} \\
& =\sum_{n=1}^{N} \lambda^{N-n} \mid M y(n)-\mathbf{w}^{H}\left(e^{-j \phi_{1}} \mathbf{a}_{1}(n)+\cdots+\left.e^{-j \phi_{M}} \mathbf{a}_{M}(n)\right|^{2}\right. \\
& =\sum_{n=1}^{N} \lambda^{N-n}\left|M y(n)-\mathbf{w}^{H} \mathbf{a}(n)\right|^{2}
\end{aligned}
$$

where

$$
\mathbf{a}_{m}(n)=\left[\begin{array}{c}
a_{m}(n), a_{m}(n)\left|a_{m}(n)\right|^{2}, \cdots, a_{m}(n)\left|a_{m}(n)\right|^{2(Q-1)}, a_{m}(n-1), \\
\cdots, a_{m}\left(n-L_{p}\right)\left|a_{m}\left(n-L_{p}\right)\right|^{2(Q-1)}
\end{array}\right]^{T}
$$

$\lambda \in[0,1]$ is a forgetting factor, and $\mathbf{a}(n)=e^{-j \phi_{1}} \mathbf{a}_{1}(n)+\cdots+e^{-j \phi_{M}} \mathbf{a}_{M}(n)$. The cost function in (3) is based on the sum of $M$ errors, each of which is the difference between $m$-th PA input and the post-distorter output. Finding $\mathbf{w}$ that minimizes $\varepsilon$ is the solution by considering all the PAs. The optimum $\mathbf{w}$ is found through the recursive least squares (RLS) algorithm [7], summarized in Table I.

\section{Table I. Summary of RLS Algorithm}

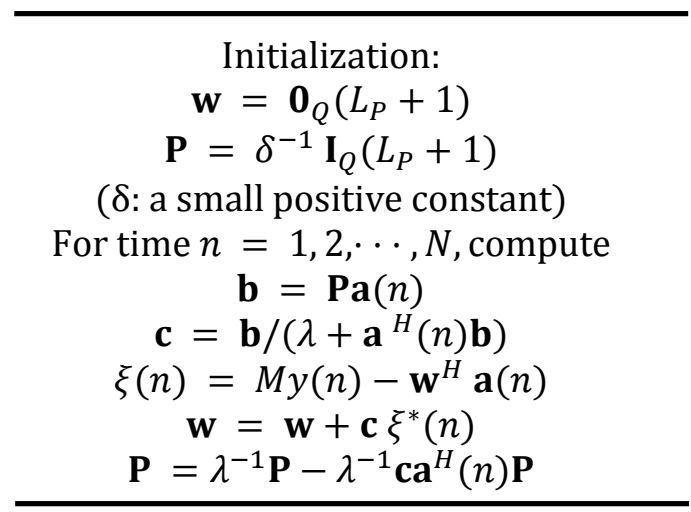

\section{Computer Simulation}

To verify the performance of the proposed DPD, we carried out computer simulation with MATLAB. The details of the simulation environments are as follows. The transmitted signal is Long Term Evolution (LTE) Downlink signal with $20 \mathrm{MHz}$ bandwidth. Saleh model, analytical formulation of a general structure for nonlinear distortion, is used for PA in the simulation [8]. This formulation is shown as follows:

$$
\mathrm{y}(\mathrm{n})=x(n) \times \frac{\alpha K_{1}}{1+K_{2}|x(n)|^{2}} e^{i \frac{\alpha K_{3}|x(n)|^{2}}{1+K_{4}|x(n)|^{2}}}
$$


We introduce $\alpha$ to change the PA characteristics among $M$ PAs. The number of antennas is $4(M=4)$, and $\alpha$ s are in turn $1,1.04,1.08$ and 1.12 , respectively.

We assume that the conventional DPD works for only one PA among four. Specifically, in the simulation, the DPD characteristics are obtained from the first PA's feedback. Therefore, the linearization performance is expected to be good for the first PA but bad for the other PAs. Figure 2 and Figure 3 show the AM/AM (amplitude-to-amplitude modulation) and AM/PM (amplitude-to-phase modulation) characteristics after applying the conventional DPD and proposed DPD, respectively. The Figures (a), (b), (c) and (d) are AM/AM of the first PA and AM/PM of the first PA, AM/AM of the third PA and AM/PM of the third PA, respectively. Each figure has four curves: DPD characteristics (blue); PA characteristics without DPD (red); PA characteristics with DPD (green). According to Figures 2 and 3, both methods can linearize the PAs and it is not easy to determine which method is better than the other. To see the difference clearly, the PA output spectrums are compared.
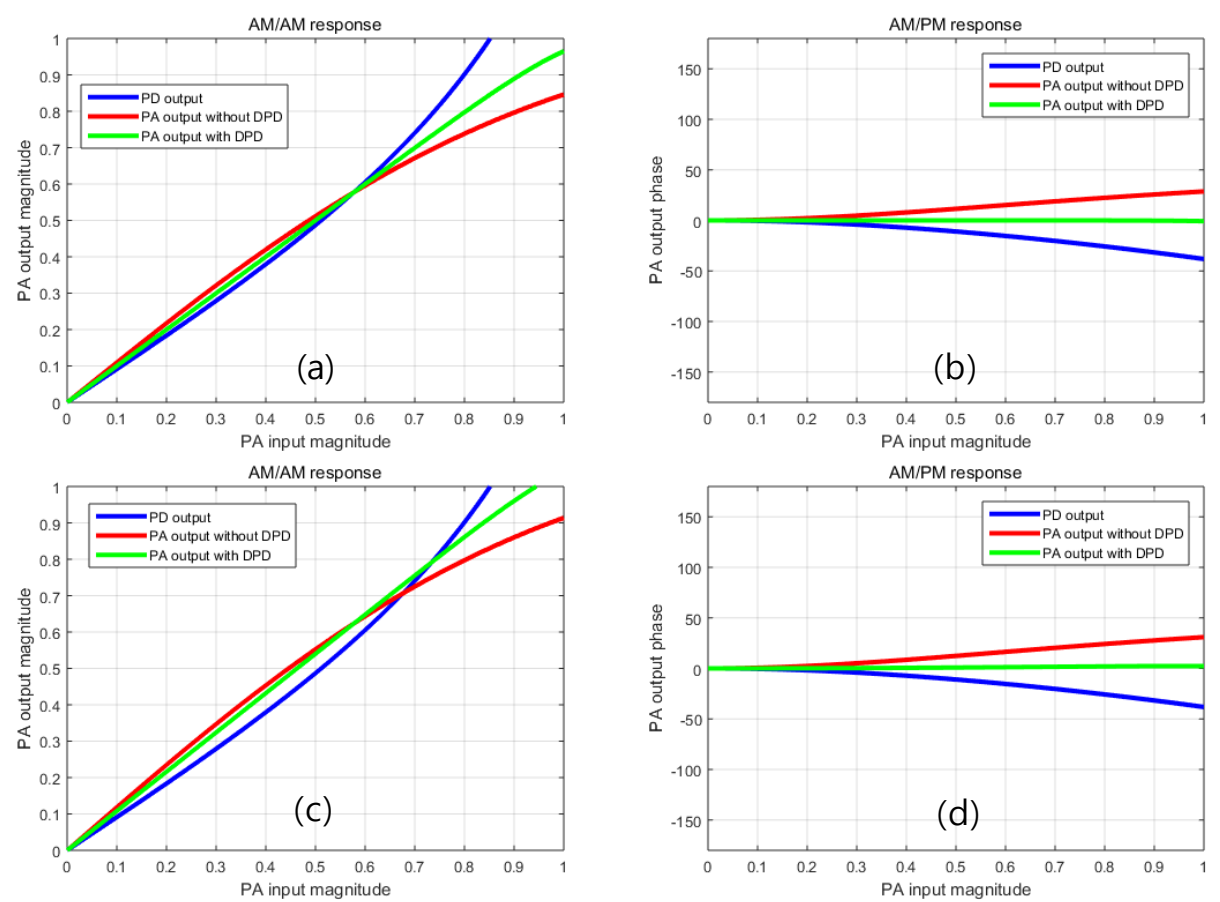

Figure 2. AM/AM \& AM/PM Response for Conventional DPD

(a): AM/AM at $1^{\text {st }} P A$; (b): AM/PM at $1^{\text {st }} P A ;(c): A M / A M$ at $3^{\text {rd }} P A ;(d): A M / P M$ at $3^{\text {rd }} \mathrm{PA}$ 

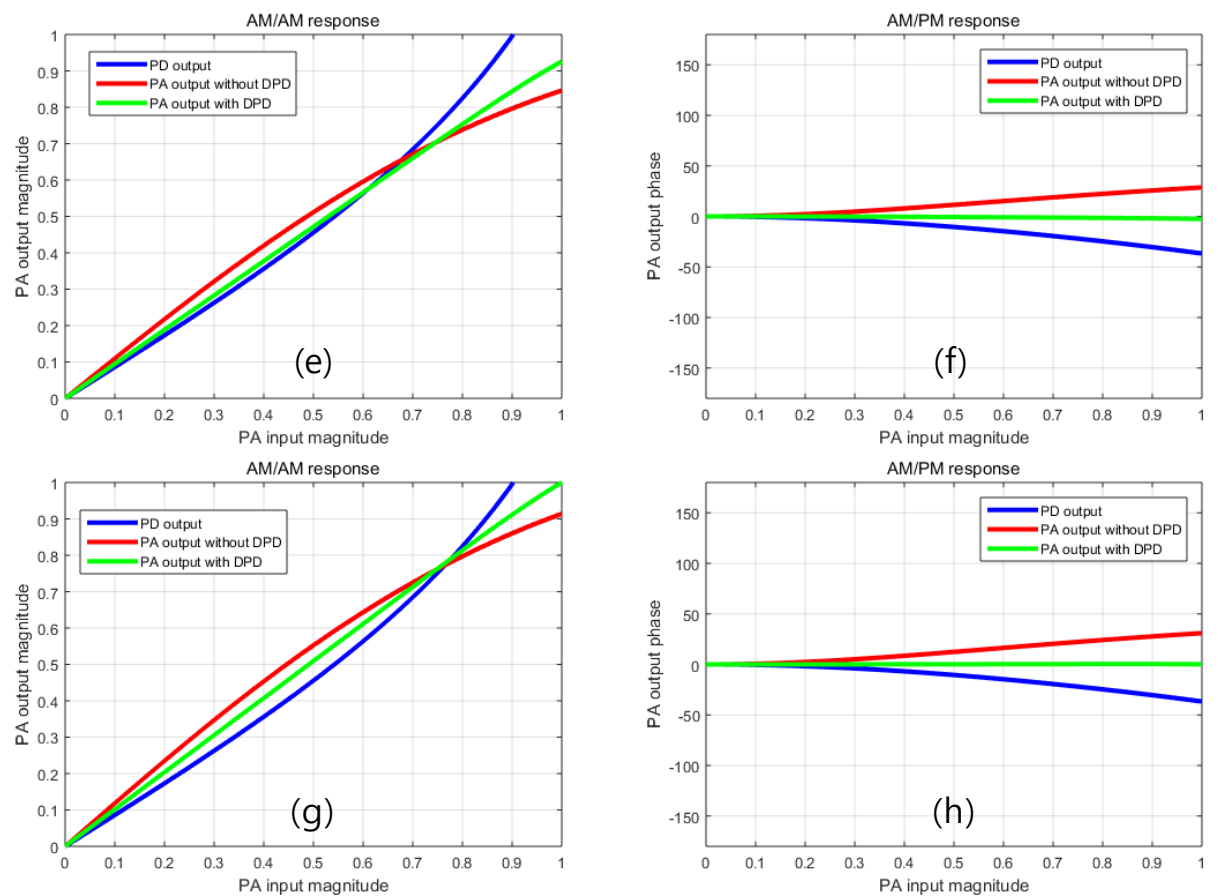

Figure 3. AM/AM \& AM/PM response for proposed DPD (a): AM/AM at $1^{\text {st }}$ PA; (b): AM/PM at $1^{\text {st }}$ PA; (c): AM/AM at $3^{\text {rd }}$ PA; (d): AM/PM at $3^{\text {rd }}$ PA

Figures 4 and 5 show the output spectrum of the PAs. The graphs are as follows: (a) DPD input (ideal signal); (b) PA output without DPD; (c) PA output with conventional DPD; (d) PA output with proposed DPD. Comparing (a) and (b), it can be seen that the spectral regrowth occurred by the non-linear PAs. Since the conventional DPD is designed to linearize the first PA only, ignoring the other PAs, the conventional DPD can linearize the first PA better than the proposed one. However, the proposed DPD performs better at the rest of the PAs. Table II summarizes the spectral regrowth reductions of DPD techniques. Those results indicate that the proposed DPD is effective for the analog beamforming systems when the PA characteristics are quite different across the PAs.
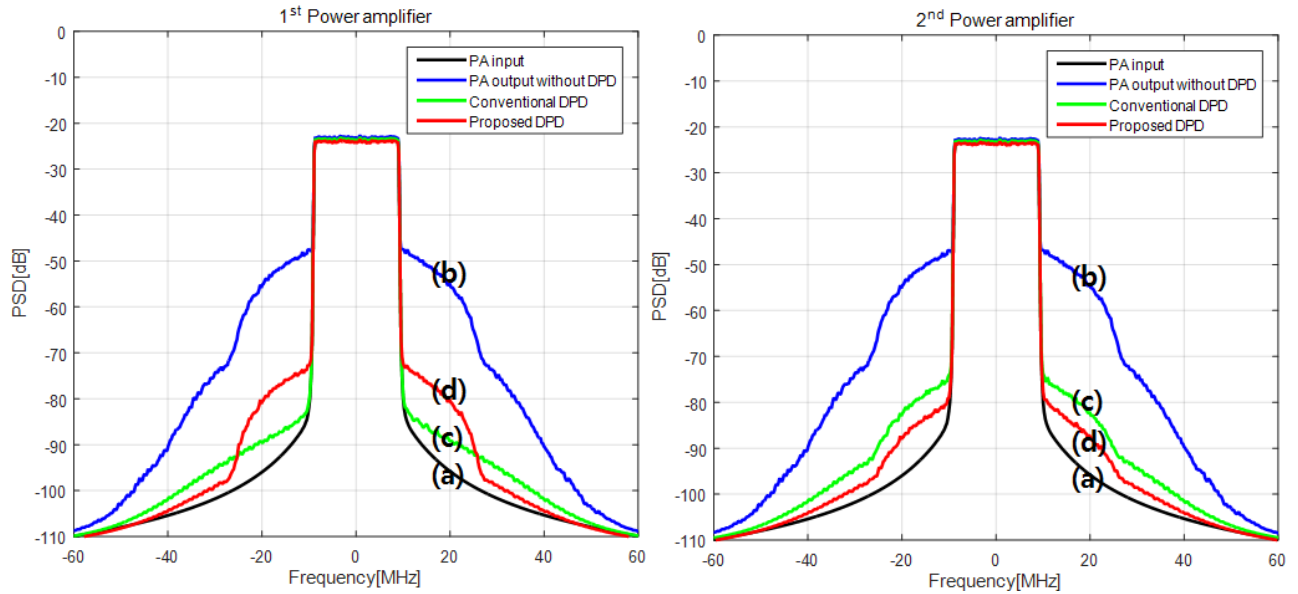

Figure 4. Spectrums at $1^{\text {st }}$ and $2^{\text {nd }}$ PA Outputs 

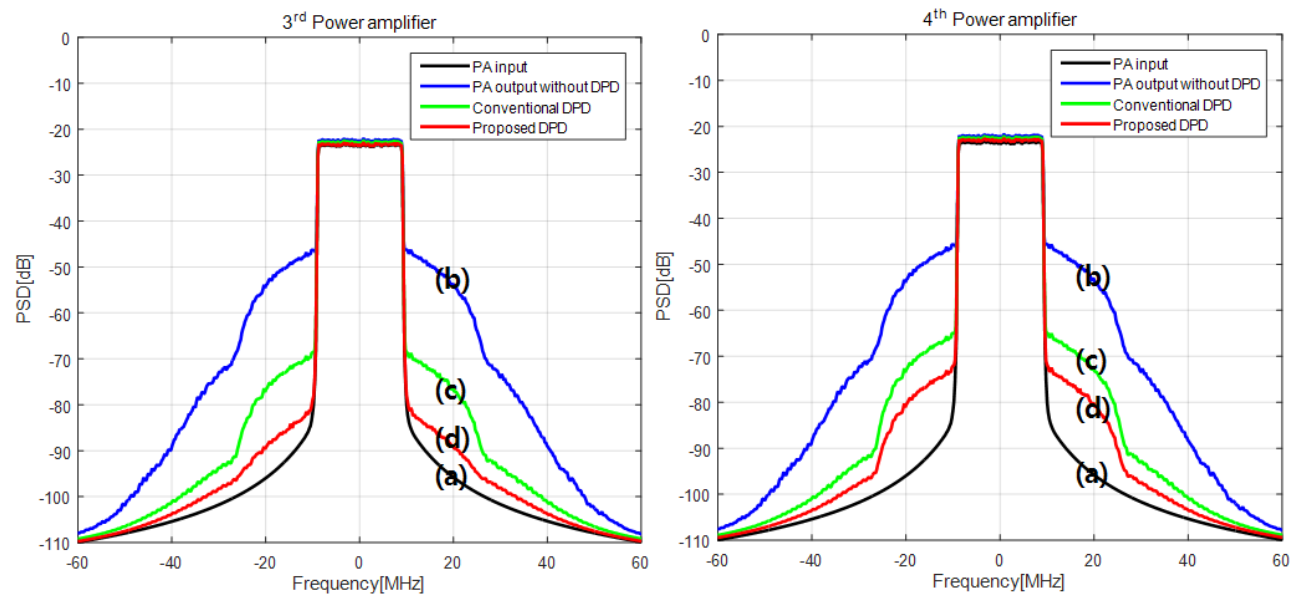

Figure 5. Spectrums at $3^{\text {rd }}$ and $4^{\text {th }}$ PA Outputs

Table II. Performance of Spectral Regrowth Reduction

\begin{tabular}{|c|c|c|}
\hline & Proposed DPD $(\mathrm{dB})$ & Conventional DPD $(\mathrm{dB})$ \\
\hline $1^{\text {st }} \mathrm{PA}$ & 25 & 37 \\
\hline $2^{\text {nd }} \mathrm{PA}$ & 34 & 28 \\
\hline $3^{\text {rd }} \mathrm{PA}$ & 35 & 23 \\
\hline $4^{\text {th }} \mathrm{PA}$ & 28 & 20 \\
\hline
\end{tabular}

\section{Experiment Results}

The proposed DPD is verified through experiment with commercial PAs. Figure 6 shows the experimental setup. We used long term evolution (LTE) downlink signals with $20 \mathrm{MHz}$ bandwidth, the same as in the simulation. The number of antennas is $2(M=2)$. The signal generator (E4438C by Agilent) converts the digital waveform into analog waveform. The center frequency of the waveform is $2605 \mathrm{MHz}$. The analog waveform is divided into two signals by the power divider, and supplied to commercial PAs. The PA has $48.5 \mathrm{~dB}$ gain. To implement phase shifters, we used two cables with different lengths. Our experiment environment provides 122 degree phase difference between two paths. The PA output is $40 \mathrm{dBm}$. The BPFs at the PA outputs attenuate the harmonics. The BPF output are directly sampled by the digital oscilloscope (TDS7254 by Tektronix) and supplied to the PC. At the initial phase, the DPD is bypassed and the DPD parameters are obtained from the Tx waveform and the PA output waveform by the adaptive algorithm described in Table I.

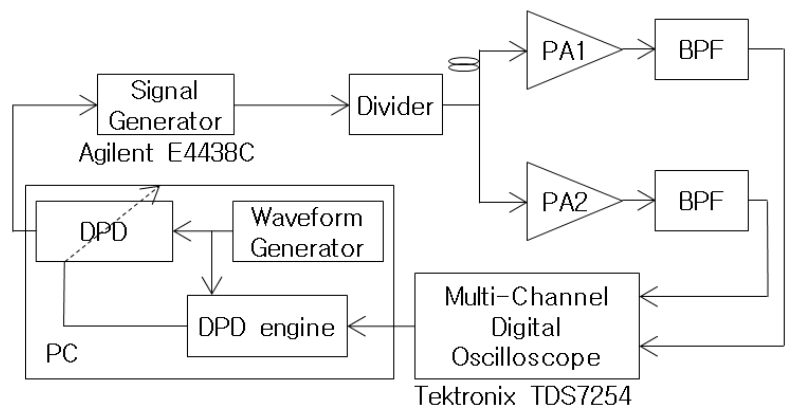

Figure 6. Digital Pre-Distortion Structure In Analog Beamforming Systems 
Learning curves for the mean square errors (MSEs) are examined first. Figure 7 shows the learning curve for the proposed DPD. According to the results, the adaptive algorithms converge within 300 samples, and the MSE after convergence is around $10^{-4}$.

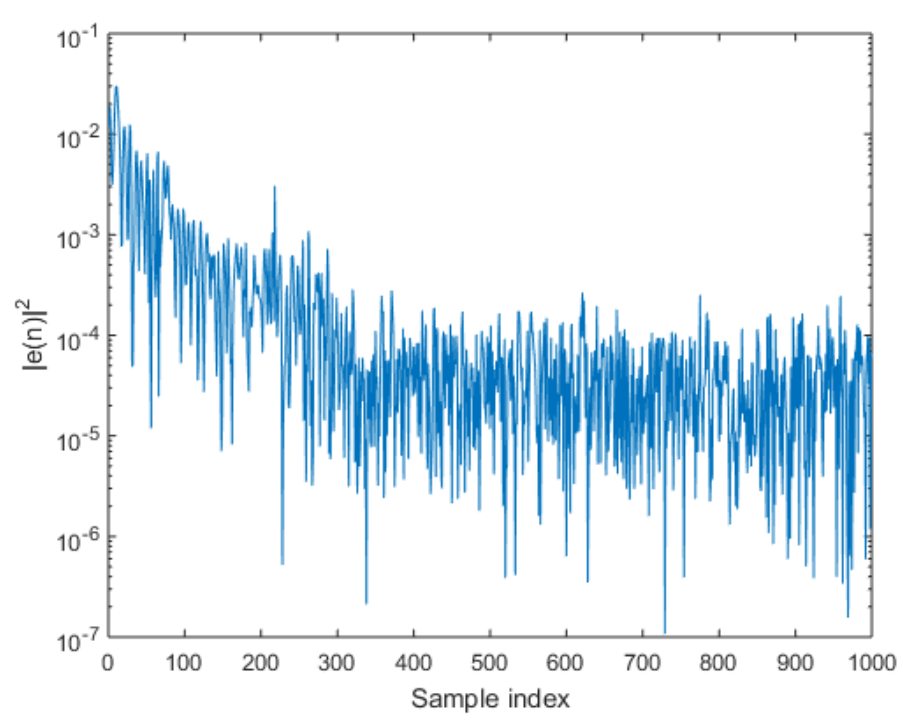

Figure 7. Learning Curves with Proposed DPD

Figures 8 and 9 show the AM/AM and AM/PM characteristics for the first PA and the second PA, respectively. The red curves represent the PA characteristics without DPD and the blue curves represent the DPD characteristics. It seems that the PD characteristics are the inverse characteristics of the PA.
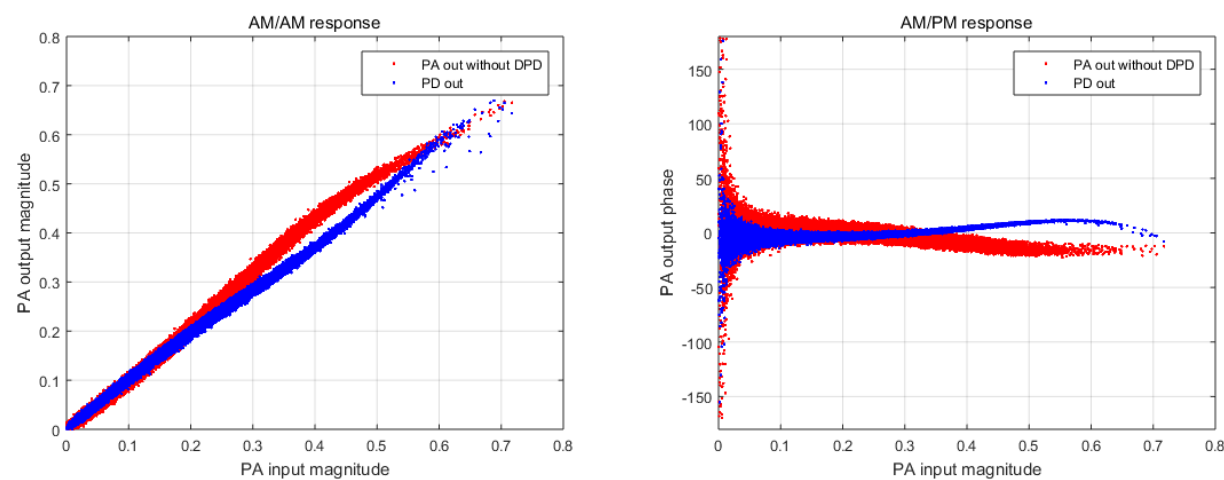

Figure 8. AM/AM and AM/PM Response at $1^{\text {st }} \mathrm{PA}$ 

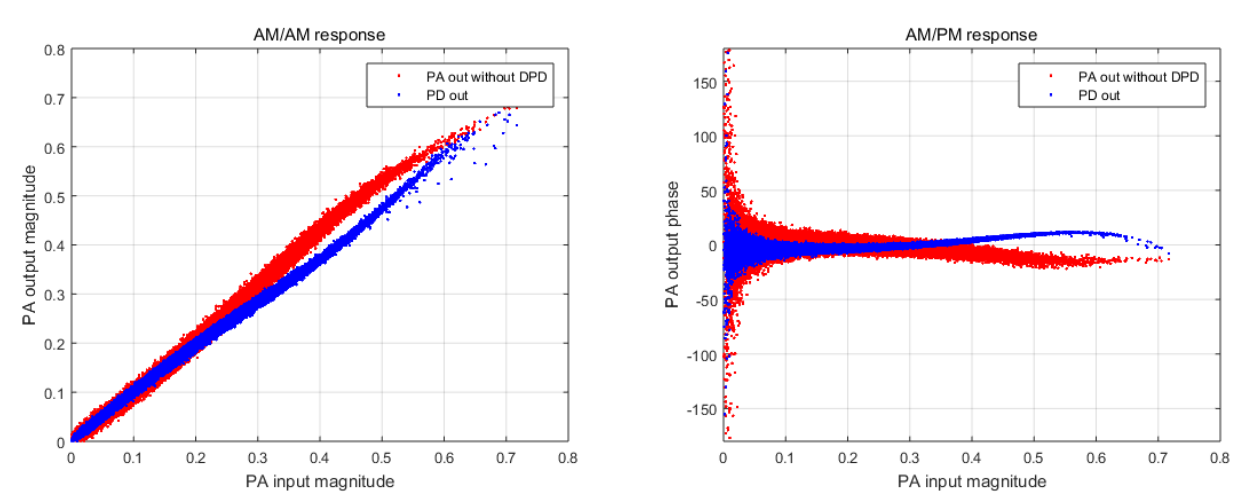

Figure 9. AM/AM and AM/PM Response at $2^{\text {nd }} P A$

To examine the linearization performance in detail, the PA output spectrums are observed as shown in Figures 10 and 11. The curves represent as follows: PA output without DPD (blue); PA output with conventional DPD (red); PA output with proposed DPD (green). Table III summarizes how much spectral regrowth can be reduced by the DPDs.

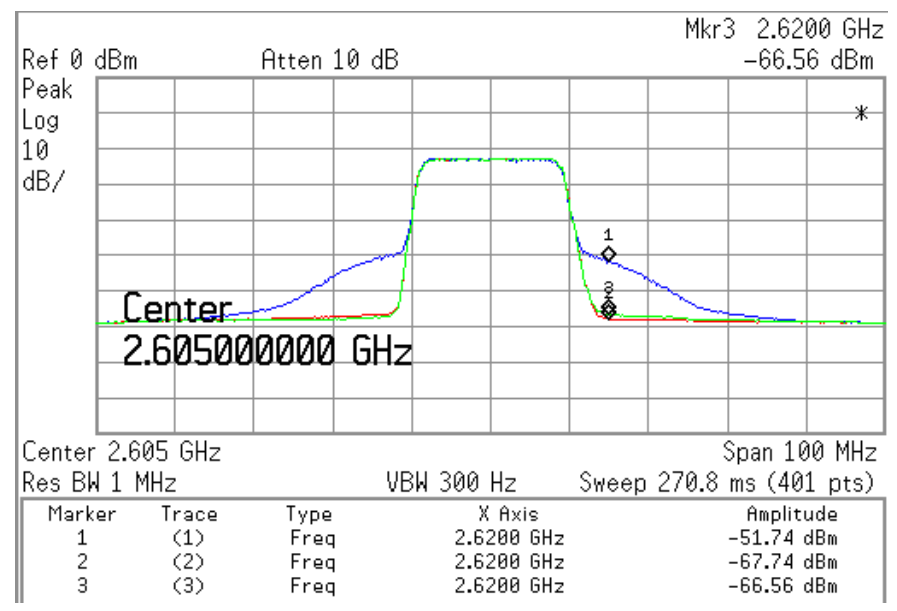

Figure 10. Spectrum at $1^{\text {st }}$ PA Output

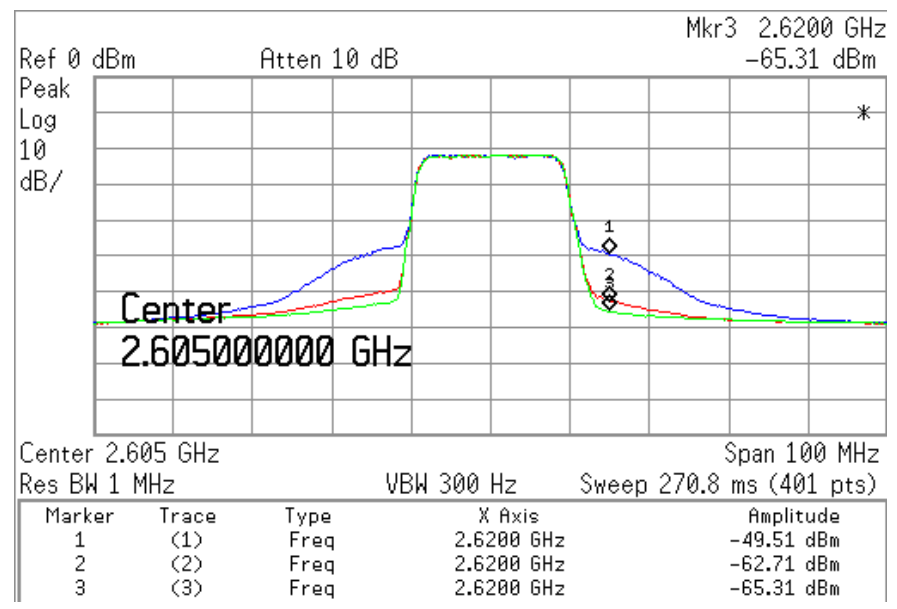

Figure 11. Spectrum at $2^{\text {nd }}$ PA Output 
Table III. Performance to Reduce Spectral Regrowth

\begin{tabular}{|c|c|c|}
\hline & Proposed DPD $(\mathrm{dB})$ & Conventional DPD $(\mathrm{dB})$ \\
\hline 1st PA & 14.82 & 16 \\
\hline 2nd PA & 15.8 & 13.2 \\
\hline
\end{tabular}

According to the results, the conventional DPD at the PA shows the best spectrum. The proposed DPD reduces about $14.8 \mathrm{~dB}$ while the conventional DPD reduces $16 \mathrm{~dB}$. On the contrary, the conventional DPD at 2 nd PA reduces the spectral regrowth by only $13.2 \mathrm{~dB}$ while the proposed one reduces $15.8 \mathrm{~dB}$. Those results indicate that the proposed DPD can successfully linearize the two PAs simultaneously.

\section{Conclusion}

A DPD technique the analog BF system was proposed in this paper. Via computer simulation and experiment, it was verified that the proposed DPD could successfully linearize the multiple PAs while the conventional DPD has a large discrepancy in linearization performance across the PAs. The result indicates that the proposed DPD can be applied to analog BF systems.

\section{Acknowledgments}

This work was supported by ICT R\&D Program of MSIP/IITP [14-911-01-001, Development of quasi-millimeter-wave channel-adaptive antennas and transceivers.

\section{References}

[1] M. Mohaisen, "A Review of Fixed-Complexity Vector Perturbation for MU-MIMO”, Journal of Information Processing Systems, 11(3), (2015), 354-369.

[2] J. An and S. Kim, "Spatial Multiplexing Receivers in UWB MIMO Systems based on Prerake Combining", Journal of information and communication convergence engineering, vol. 9, no. 4, (2011), pp. 385-390.

[3] V. Venkateswaran and A.-J. Van Der Veen, "Analog beamforming in MIMO communications with phase shift networks and online channel estimation", IEEE Transactions on Signal Processing, vol. 58, no. 8, (2010), pp. 4131-4143.

[4] A. J Paulraj, “An overview of MIMO communications-a key to gigabit wireless”, Proceedings of the IEEE, vol. 92, no. 2, (2004), pp. 198-218.

[5] S. Choi and E-R. Jeong, "Digital predistortion based on combined feedback in MIMO transmitters", IEEE Comm. Lett., vol. 16, no. 10, (2012), pp. 1572-1575.

[6] S. Choi, E.-R. Jeong and Y. H. Lee, "A direct learning structure for adaptive polynomial-based predistortion for power amplifier linearization", Vehicular Technology Conference, 2007. VTC2007-Spring. IEEE 65th. IEEE, (2007).

[7] S. Choi, E-R. Jeong and Y. H. Lee, "Adaptive predistortion with direct learning based on piecewise linear approximation of amplifier nonlinearity”, IEEE J. Sel. Topics Signal Process., vol. 3, no. 3, (2012), pp. 397-404.

[8] A. A. M. Saleh, "Frequency-independent and frequency dependent nonlinear models of TWT amplifiers", IEEE Trans. Commun., vol. COM-29, no. 11, (1981), pp. 1715-1720.

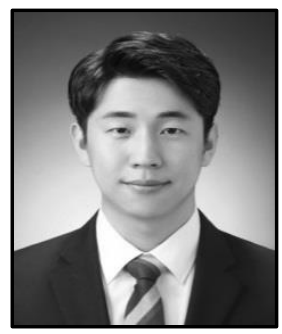

Haeng-Bok Kil, he received his B.S. degree in the Department of Radio Wave Engineering from Hanbat National University, Daejeon, Korea, in 2016. He is currently pursuing his Master's Degree in the Department of Radio Wave Engineering from Hanbat National University, Daejeon, Korea. His research interests are in the areas of digital signal processing, pre-distortion, and modem design. 


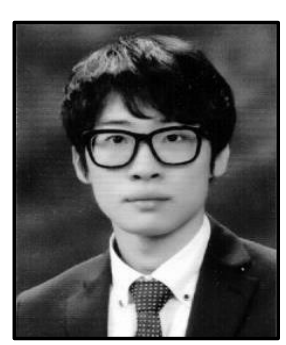

Kwang-Pyo Lee, she received his B.S. degree in the Department of Radio Wave Engineering from Hanbat National University, Daejeon, Korea, in 2015. He is currently pursuing his Master's degree in the Department of Radio Wave Engineering from Hanbat National University, Daejeon, Korea. His research interests are in the areas of digital signal processing, pre-distortion, and modem design.

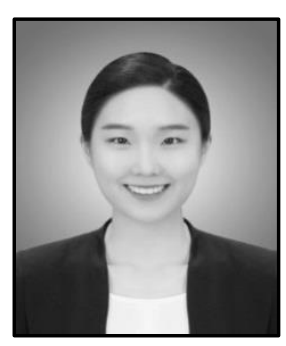

Sung-Mi Jo, she received her B.S. degree in the Department of Radio Wave Engineering from Hanbat National University, Daejeon, Korea, in 2016. She is currently pursuing her Master's degree in the Department of Radio Wave Engineering from Hanbat National University, Daejeon, Korea. His research interests are in the areas of digital signal processing, pre-distortion, and modem design.

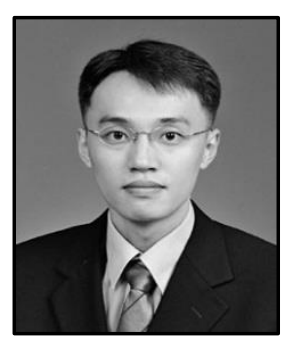

Eui-Rim Jeong, he received his B.S., M.S., and Ph.D. degrees in the Department of Electrical Engineering from Korea Advanced Institute of Science and Technology (KAIST), Daejeon, Korea, in 1995, 1997, and 2001, respectively. He has been an associate professor in Department of Information and Communication Engineering, Hanbat National University, since 2009. His research interests are in the areas of communication signal processing, predistortion, and modem design. 